\title{
ARENA - Plataforma para Experimentação de Sistemas Multi-Robôs em Ambientes Industriais
}

\author{
Marcelo Limeira $^{1}$, André Schneider de Oliveira ${ }^{1}$ \\ ${ }^{1}$ Universidade Tecnológica Federal do Paraná (UTFPR) \\ Av. Sete de Setembro, 3165 - Curitiba - PR - Brasil \\ limeira@alunos.utfpr.edu.br, andreoliveira@utfpr.edu.br
}

\begin{abstract}
This work presents ARENA - a platform for experimenting with advanced behaviors in smart warehouses. The proposed method consists of reproducing a reduced-scale warehouse managed by a group of autonomous forklifts, represented by a swarm of microrobots compatible with the ROS (Robot Operating System) architecture. Augmented reality (AR) is used to improve the platform's resources, allowing it to present features that are unfeasible due to the reduced size of its elements or due to the cost, such as the movement of goods and the implementation of certain sensors.
\end{abstract}

Resumo. Este trabalho apresenta a ARENA - uma plataforma para experimentação de comportamentos avançados em armazéns inteligentes. O método proposto consiste na reprodução de um armazém, em escala reduzida, gerenciado por um grupo de empilhadeiras autônomas, representadas por um enxame de micro robôs compatíveis com a arquitetura ROS (Robot Operating System). A realidade aumentada ( $R A$ ) é empregada para aprimorar os recursos da plataforma, permitindo que essa apresente características inviabilizadas pelo tamanho reduzido de seus elementos ou em função do custo, como a movimentação de mercadorias e a implementação de determinados sensores.

Dissertação submetida ao CTDR 2021 - Data da defesa: 10/10/2019. Orientador: André Schneider de Oliveira.

Este trabalho foi contribuição direta para dois Congressos Internacionais [Limeira et al. 2019, Limeira et al. 2020] e três periódicos internacionais [Limeira et al. 2021, Kalempa et al. 2020, Piardi et al. 2019].

\section{Introdução}

A $4^{\text {a }}$ revolução industrial, também conhecida como indústria 4.0, implementou uma série de avanços tecnológicos, que transformaram, de forma significativa, todos os aspectos da atividade industrial. Essa iniciativa tem como principal objetivo a digitalização dos processos, transformando as fábricas tradicionais em fábricas inteligentes. De acordo com [Kagermann et al. 2013], a Indústria 
4.0 pode ser vista como uma combinação de Tecnologias de Informação e Comunicação (TIC) e Sistemas Ciber-Físicos (CPS - Cyber-Phisical Systems), no ambiente de fabricação, para otimizar o processo de produção, por meio da interação de agentes dinâmicos interconectados. Um segmento da indústria que tem utilizado esses recursos, de forma especialmente eficiente, tem sido os armazéns inteligentes, como ocorre na Amazon [Li and Liu 2016], Ocado [Mason 2019] e DHL [Lee 2018]. Nesses, o SMR (sistemas multi-robôs) são implantados para realizar a movimentação das mercadorias, de forma mais eficiente, otimizando tempo e recursos. Esses conceitos apresentam um potencial extraordinário nas operações de armazéns automatizados e têm atraído, cada vez mais, a atenção da comunidade acadêmica.

Apesar de todo o entusiasmo, atualmente, há limitações em termos de aplicações reais. A maior parte dos trabalhos desenvolvidos nessa área é elaborada e validada apenas em simuladores virtuais, como: Webots [Michel 1998], V-REP [Rohmer et al. 2013], Gazebo [Koenig and Howard 2004] e MORSE [Echeverria et al. 2012]. Esses simuladores apresentam a vantagem de permitir a reprodução de diferentes ambientes e cenários, de forma muito prática, com a utilização de elementos virtuais. Contudo, esses possuem desvantagens que os tornam inadequados para o desenvolvimento de aplicações reais, como a impossibilidade de verificar o funcionamento de todo o hardware que compõe o sistema (roteadores, sensores, motores, controladores e demais componentes do robô, entre outros). Outra desvantagem presente nesses softwares é que esse não reproduzem, de forma fiel, todas as condições encontradas em ambientes reais, como: iluminação, atrito, imprecisão de sensores etc.

\subsection{Objetivos}

Com base nos fatores expostos até aqui e, analisando abordagens propostas na literatura, esse trabalho propõe uma abordagem para a produção e experimentação de sistemas multi-robôs em ambientes industriais. O conceito da ARENA é apresentado e se refere a uma plataforma híbrida, na qual elementos reais e virtuais serão utilizados para representar, em escala reduzida, o cenário e todas as operações de um armazém inteligente, gerenciado por robôs móveis. A plataforma utiliza pequenos robôs reais (Wsbot [Limeira et al. 2019]), que apresentam praticamente todas as características presentes em robôs utilizados na indústria (sistema de locomoção, autonomia, sistema de rastreamento, sensor de obstáculos etc.). A utilização de elementos virtuais, através da RA, será utilizada para a construção de características do cenário, para a representação gráfica de dados dos sensores e para reproduzir condições que poderiam ser inviabilizadas por questão orçamentária ou em função do tamanho reduzido de toda a estrutura (sensores, movimentação de mercadoria etc.). O fato de toda a estrutura ser representada em escala reduzida, possibilita que a arena seja implementada, em um laboratório, permitindo acesso a todos os recursos e ferramentas presentes nesse ambiente. 


\section{Estado da Arte}

Os SMR apresentam amplas aplicações e imenso potencial. No entanto, desenvolvê-los é uma tarefa extremamente complexa em decorrência da grande quantidade de sistemas operando, de forma paralela. Desse modo, existe uma grande quantidade de potenciais fontes de falhas. Além de todos os problemas, que podem ocorrer com um único robô (falha mecânica, mau funcionamento de sensores ou falhas no software), existe uma série de outros elementos passíveis de falha na plataforma (sistema de rastreamento, elementos virtuais, câmeras etc.). A seguir serão apresentadas algumas abordagens de técnicas, de ferramentas e de plataformas propostas para auxiliar o desenvolvimento destes sistemas.

Em [Antoun et al. 2016] é proposto um sistema que utiliza uma superfície de vidro chamada Kilogrids, por meio da qual os Kilobot (robôs) se movimentam. O Kilogrid possui a capacidade de configuração do ambiente, por meio de células coloridas por LEDS, dispostos em uma configuração de 64 células divididas em 8 linhas e 8 colunas. Foram, ainda, desenvolvidos sensores virtuais visando superar as limitações impostas pelos Kilobots como, por exemplo, a capacidade de determinar sua localização. Já em [Reina et al. 2017], é apresentado um sistema denominado ARK (Augmented Reality for Kilobots). Este sistema utiliza quatro câmeras na parte superior da estrutura para realizar o rastreamento e controle, além de utilizar a realidade aumentada para projetar cenários e, assim, permitir que os robôs possam interagir com esses. Em [Reina et al. 2015], é proposto um projeto similar ao anterior chamado de ARGoS. Este projeto conta com um sistema de rastreamento, um simulador e robôs swarms e-pucks. O sistema de rastreamento utiliza marcadores visuais sobre os robôs (ARtag [Zhang et al. 2002]), que são reconhecidos por câmeras dispostas na parte superior da estrutura. Também foram adicionados recursos de realidade aumentada para a projeção de determinadas características no ambiente e nos robôs, cujo foco principal é o desenvolvimento de sensores virtuais.

Provavelmente, o simulador mais popular e conhecido seja o Robotarium [Pickem et al. 2017] mantido pela Georgia Institute of Technology. Este simulador, que também é um laboratório, está operando, em sua segunda versão, que possui mais de cem robôs sobre uma mesa de 240 x $365 \mathrm{~cm}$. Os robôs, chamados de GRITSBots, apresentam um sistema de rastreamento por marcação visual (AR-Tags) e comunicação infravermelho. É possível, ainda, projetar objetos virtuais por meio da realidade aumentada.

Com base na escassez de trabalhos que apresentem contribuições para a modernização da indústria, por meio da robótica móvel, é bastante valiosa a construção de uma plataforma que possa servir de base para a produção, experimentação e validação de diferentes abordagens relacionadas a essa tecnologia. A possibilidade de superar os diversos fatores que, inegavelmente, dificultam a realização destes projetos, poderá proporcionar um aumento na 
quantidade e na qualidade destes trabalhos.

\section{A Arena}

A ARENA (figura 1) é um novo conceito para a experimentação ativa em armazéns inteligentes, cujo objetivo é promover as características reais do chão de fábrica. Essa foi inspirada em um armazém real, localizado no Brasil. Dessa maneira, todos os setores como manutenção, recarga de robôs, entrada e saída de produtos, de armazenagem, de triagem, entre outros, bem como toda a sua dinâmica de operação, foram fielmente reproduzidos no modelo. Na próxima seção será apresentada a estrutura do armazém utilizado como modelo, em seguida, será exposta a forma como os recursos da ARENA serão utilizados para representar todas as suas características e operações.

Figura 1. ARENA

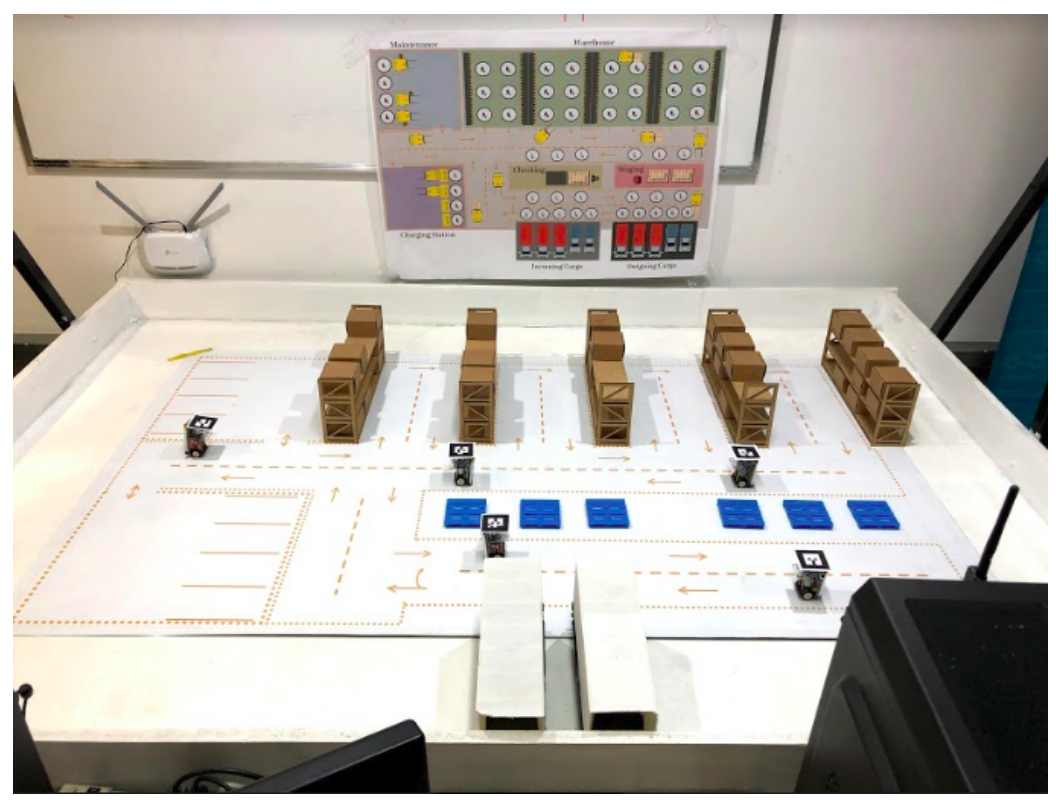

Fonte: Autoria própria.

\subsection{Estrutura da ARENA}

A abordagem da ARENA é uma composição especialmente projetada para avaliar novos métodos com os requisitos da Indústria 4.0, como robótica avançada, IOT (internet das coisas), inteligência artificial, Realidade Aumentada (AR), e comunicação M2M (máquina a máquina).

O projeto ARENA está organizado em quatro eixos fundamentais - real, virtual, camadas e sensores. O eixo Real é a infraestrutura do armazém, em que os componentes físicos (caminhões, paletes, prateleiras) são embutidos em escala reduzida (maquetes). A AR apresenta novas possibilidades com a adição de elementos virtuais, no eixo Virtual. Assim, pequenos robôs podem lidar com grandes caixas, executando as ações de entrada e saída de carga. Novos agentes virtuais podem ser adicionados ao sistema para uma avaliação, 
em vários cenários, com diferentes tipos de empilhadeiras virtuais, ou para a análise da interação homem-robô com operadores virtuais. O eixo Sensor está relacionado aos recursos de percepção global e local. Os pequenos robôs são dotados com sensores LRF virtual [Limeira et al. 2021], o que os capacita a identificar obstáculos.

A infraestrutura é montada sobre uma mesa grande personalizada, que suporta componentes estáticos e dinâmicos, com a dimensão de 1,4 $\mathrm{m}$ de comprimento e 2,1 $\mathrm{m}$ de largura. Esse suporte é composto de madeira e está coberto por uma lona de vinil, que é plotada com a planta da indústria usada como modelo, ou seja, o armazém. Ao lado da mesa há uma estrutura metálica com uma altura de 1,6 m, utilizada para suportar câmeras e os refletores. Duas câmeras RGB e uma câmera RGB-D são empregadas na ARENA para representar toda a funcionalidade necessária para o pleno desenvolvimento do sistema. Uma câmera RGB é dedicada ao rastreamento de robôs por meio do processamento de imagens, localizando a posição e a orientação das AR-Tags fixadas em cima dos pequenos robôs móveis, os WsBots. Outra câmera RGB é utilizada para $A R$, em que camadas virtuais são adicionadas à imagem original para obter uma experiência mais realista e imersiva. A câmera RGB-D apresenta os sensores virtuais no WsBots, para obter os mesmos recursos do LRF usados em empilhadeiras reais.

Outro componente estático importante presente na ARENA é a estação de carregamento. Essa é composta por quatro transmissores de carga sem fio, que possuem um circuito eletrônico e uma bobina para transmitir a energia a uma bobina receptora acoplada ao robô. Assim, é possível carregar os robôs, de forma autônoma, sem a necessidade de conectá-los, manualmente, à energia ou de desligar o sistema do robô. Elementos reais do armazém são adicionados para obter a percepção real do ambiente, no qual são introduzidas prateleiras feitas de madeira e projetadas por um CNC (Computer Numeric Control - máquina de usinagem) e paletes impressos em impressora 3D. A zona de carga de entrada e saída também é especificada por caminhões de carga de plástico. As ações das empilhadeiras são executadas pelo WsBot, que interage com os outros recursos presentes, representando uma empilhadeira de armazém real.

\subsection{Realidade Aumentada - AR}

A intenção desta plataforma é reproduzir, da forma mais fiel possível, todas as condições encontradas no ambiente apresentado, no caso, em um armazém. No entanto, em função do tamanho, em escala reduzida, da plataforma e do robô, desenvolver um dispositivo eletromecânico para o robô de forma que este pudesse erguer um objeto, realizar sua movimentação e, ainda, depositálo nas prateleiras, não seria uma tarefa trivial e, provavelmente, desnecessária. Desse modo, foram introduzidos na ARENA elementos virtuais que pudessem substituir determinadas características, ações ou condições que, por algum motivo, não puderam ser reproduzidas com elementos reais, seja por 
impossibilidade técnica ou por decisões internas do projeto. A figura 2 expõe o uso de AR em experimentação do armazém inteligente na ARENA.

Figura 2. Visão superior dos elementos AR da ARENA.

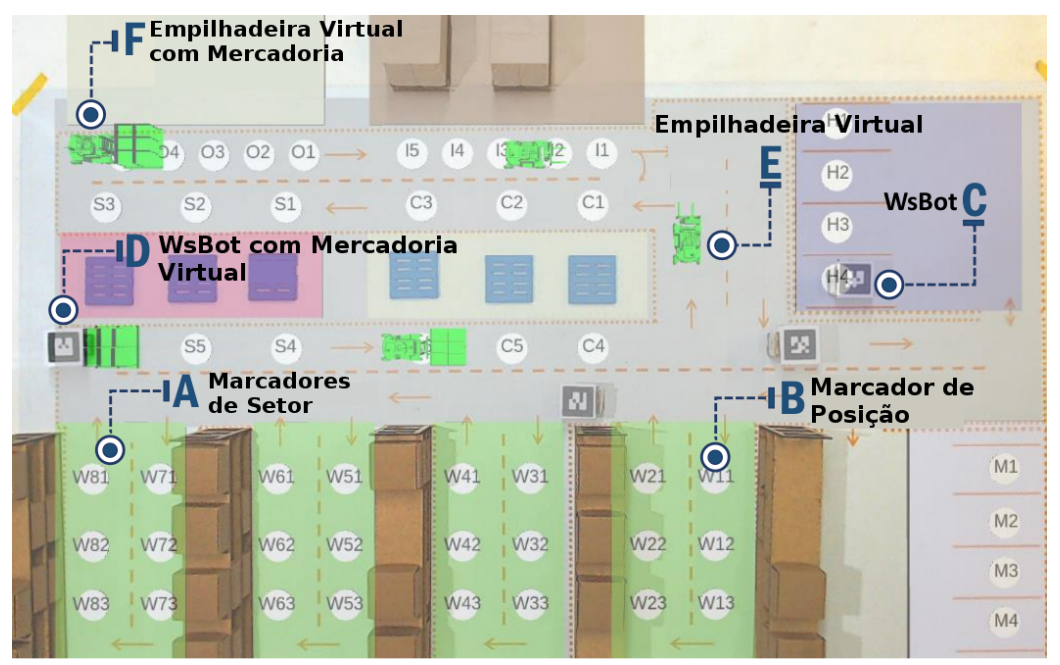

Fonte: Autoria própria.

Objetos estáticos são carregados na inicialização do sistema e têm suas posições já definidas. O marcador de posição do mapa é usado para determinar posições úteis no armazém (por exemplo, posicionamento para carregar robôs ou colocar/remover mercadorias de paletes), enquanto marcadores de setor são usados para destacar diferentes setores dentro do armazém ("A" e "B", respectivamente).

Considerando os componentes virtuais apresentados acima, o AR pode ser empregado para verificar diferentes situações na ARENA e, desse modo, desenvolver novas abordagens para problemas que, atualmente, são desafios em um armazém inteligente. Por exemplo, técnicas de planejamento de rota para evitar obstáculos podem ser desenvolvidas e validadas usando robôs virtuais interagindo com obstáculos reais ou até inserindo falhas aleatórias em robôs reais, como baterias esgotadas. A ARENA, ao implantar a realidade aumentada juntamente com a estrutura ROS, procura preencher a lacuna entre simulação e realidade, propondo um ambiente de teste mais dinâmico, respeitando as políticas e leis do mundo real e aplicando modelos reduzidos para validação do sistema de armazém inteligente. Portanto, a abordagem híbrida apresenta as vantagens da economia de tempo, de segurança e de baixo custo de simulação ao usar componentes virtuais, além de ter a fidelidade dos modelos reais em função de objetos estáticos e dinâmicos da ARENA.

\subsection{Arquitetura de software}

Neste trabalho, diversos pacotes desenvolvidos e disponibilizados pela comunidade ROS foram utilizados. A seguir, estes pacotes serão devidamente listados. 
- USB_Cam: Nó responsável pela publicação das informações capturadas pelas câmeras RGB em tópicos do ROS. A partir deste nó são criados dois tópicos, um para cada câmera [ROS 2019a].

- RVIZ (Ros Visualization): É um visualizador tridimensional que, entre as diversas funções, possibilita a projeção de objetos virtuais em uma imagem real [ROS 2019b].

- Ar_Track_Alvar: Pacote utilizado no sistema de rastreamento dos robôs. Este pacote estabelece uma relação entre marcadores geométricos impressos em uma superfície (tags) e um código ID, que é composto por um número inteiro, sequencial, iniciado em 0 e possui a capacidade de, mesmo utilizando uma imagem 2D, identificar as coordenadas em 3D destes marcadores identificados. Essas coordenadas são representadas por dois vetores, um linear $(\mathrm{x}, \mathrm{y}, \mathrm{z})$ e outro angular.

- RealSense2_Camera: Pacote desenvolvido pela Intel, que permite acesso às funções da câmera RGB-D utilizada neste trabalho como a PointCloud.

Além dos pacotes disponibilizados, pela comunidade ROS, houve o desenvolvimento, para este trabalho, de uma série de pacotes com diferentes funções, e essas serão apresentadas a seguir:

\subsubsection{Controle de movimentos}

O principal nó do sistema é o Swarm_Control. Esse é responsável por toda ação dinâmica do sistema, desde a execução do planejamento de trajetória, algoritmo de controle para a execução da rota planejada até a geração dos elementos virtuais dinâmicos, como as caixas e os marcadores de caminho percorrido. Este nó foi projetado para receber quatro parâmetros quando inicializado. O primeiro é o ID do robô, representado por um código numérico, o segundo é a posição de destino representada por um código alfanumérico (ex: W15), o terceiro e o quarto indicam se devem ser inseridos os elementos virtuais da caixa ligada ao robô e a marcação de caminho percorrido ("1" caso positivo). Assim que este nó é inicializado, são carregadas, em uma matriz, todas as coordenadas previamente determinadas dos pontos marcados no mapa, bem como as conexões possíveis entre estes pontos, em forma de gráfico. Ainda na inicialização, os parâmetros três e quatro são verificados e os respectivos elementos virtuais são habilitados, caso seja determinado. Após essa inicialização, é realizada a análise da posição do robô selecionado e a identificação do marcador de posição mais próximo ao robô.

O planejamento de trajetória é realizado por um algoritmo Dijkstra. Este algoritmo permite encontrar o caminho mais curto entre dois pontos em um gráfico. A partir da posição identificada do robô e a posição selecionada na inicialização do nó, é então planejada a rota que o robô deverá percorrer. Uma vez planejada a trajetória, o algoritmo de controle é inicializado. O sistema de controle proposto é baseado no método de controle PID. 


\section{Experimentos}

A experimentação da ARENA é avaliada nessa seção por meio de dois experimentos. O primeiro apresenta as ferramentas de realidade aumentada para a experimentação em armazém (sensor virtual, marcadores de posição, caixas, etc). O segundo experimento está voltado para a demonstração do controle de movimentação do WsBot dentro do armazém.

\subsection{Realidade aumentada da ARENA}

Esse primeiro experimento tem o objetivo de apresentar os recursos e ferramentas presentes na ARENA, sobretudo, os recursos relacionados à realidade aumentada. As técnicas de AR empregadas na ARENA promovem uma experimentação realista e altamente imersiva, permitindo o entendimento visual da logística do armazém, das zonas de produção e dos estados logísticos. Os elementos virtuais aprimoram a experimentação, não apenas em uma visão mais intuitiva, mas possibilitam uma análise mais abrangente de comportamentos inteligentes.

Figura 3. Efeito da AR na ARENA. (a) Visão superior sem AR. (b) Visão superior com AR.

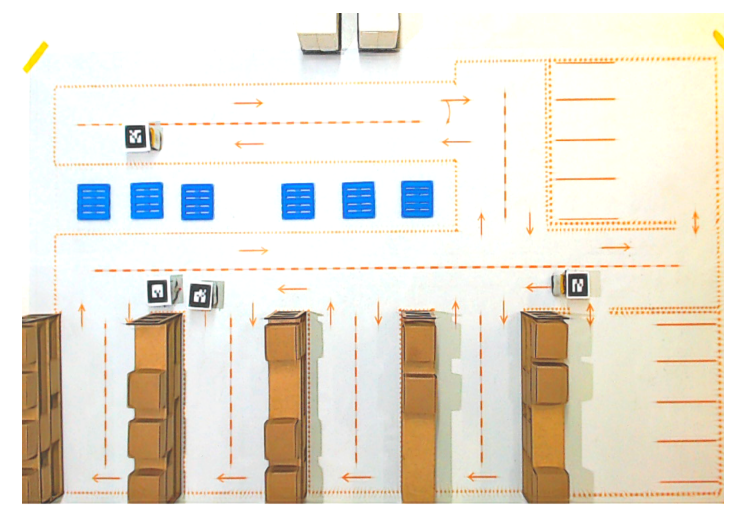

(a)

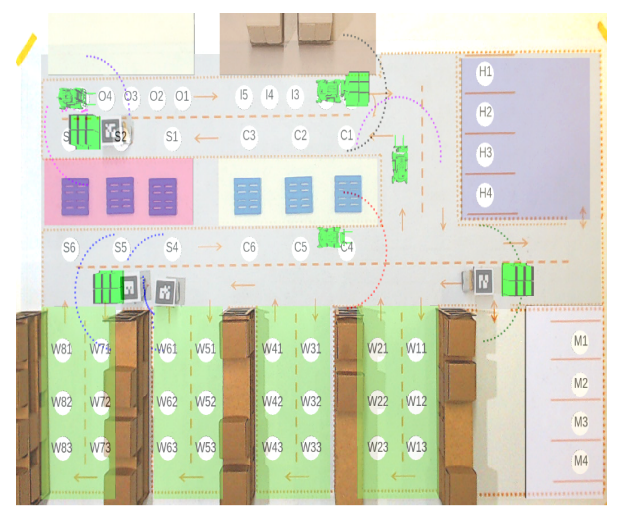

(b)

Fonte: Autoria própria.

As figuras 3a e 3b apresentam duas imagens do mesmo experimento, em que a segunda imagem conta com a presença dos elementos de AR. Neste experimento, é possível verificar a presença de praticamente todos os elementos dinâmicos criados neste projeto. Além dos já mencionados WsBots e sensores virtuais, foram adicionadas empilhadeiras e caixas virtuais. As empilhadeiras virtuais possuem as mesmas características sensoriais e de controle que os WsBots e podem ser úteis para a execução de experimentos, que necessitem de uma grande quantidade de agentes, quando não houver WsBots suficientes. É possível notar que tanto os WsBots quanto as empilhadeiras virtuais podem ou não estar movimentando as caixas virtuais. Dessa forma, é possível representar todas as possíveis condições presentes na operação do armazém. 
Figura 4. ARENA em perspectiva com AR.

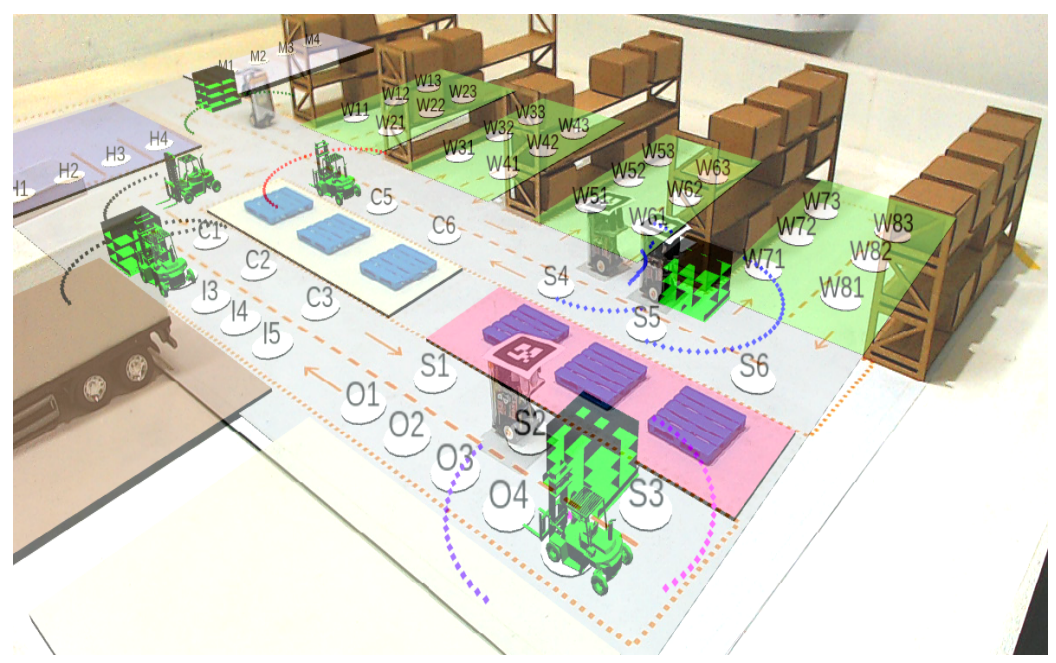

Fonte: Autoria própria.

O experimento da figura 3 b é ilustrado em uma outra perspectiva na figura 4. O sensor LRF analisa 37 pontos em um raio de 180 graus, a partir do ponto em que esse está localizado, nesse caso, no centro do robô. Para facilitar a análise, em tempo de execução dos dados fornecidos pelo sensor, pontos criados através da AR foram projetados. Cada um destes pontos em azul, presentes na imagem, representa um ponto analisado e fornecido pelo sensor, levando em consideração suas respectivas distâncias e ângulos.

\subsection{Controle de movimentos do WsBot}

Esse experimento visa demonstrar a capacidade do WsBot de se movimentar nas posições do armazém. Assim, é determinado que o WsBot 1 deve se movimentar do estado inicial $W_{23}$ para o estado intermediário $W_{7}$, enquanto o WsBot 2 deve ir do $O_{5}$ para o estado intermediário $C_{5}$. A figura 5 mostra, através de uma marcação em vermelho, o caminho percorrido pelos dois robôs em duas execuções do algoritmo. Este experimento, apesar de parecer simples, permite verificar o correto funcionamento de uma boa parte dos componentes de software e de hardware, que compõe não apenas o robô, mas toda a plataforma.

A primeira verificação, que pode ser realizada, se faz em relação ao robô em si, uma vez que uma falha em qualquer um dos seus componentes ou um erro em sua montagem, provavelmente, impediria que o robô executasse qualquer movimento. Já uma segunda análise é realizada em relação ao sistema de comunicação. Isso porque, como todas as ações de movimento realizadas são gerenciadas pelo servidor e executadas pelo software presente, em cada robô, qualquer falha na comunicação refletiria, imediatamente, nas ações executadas por esse. O sistema de rastreamento é um outro componente importante do sistema, que pode ser analisado a partir deste experimento. $\mathrm{O}$ controle do robô depende da estimativa precisa de sua posição, além da velo- 
Figura 5. Experimento de execução de rota.

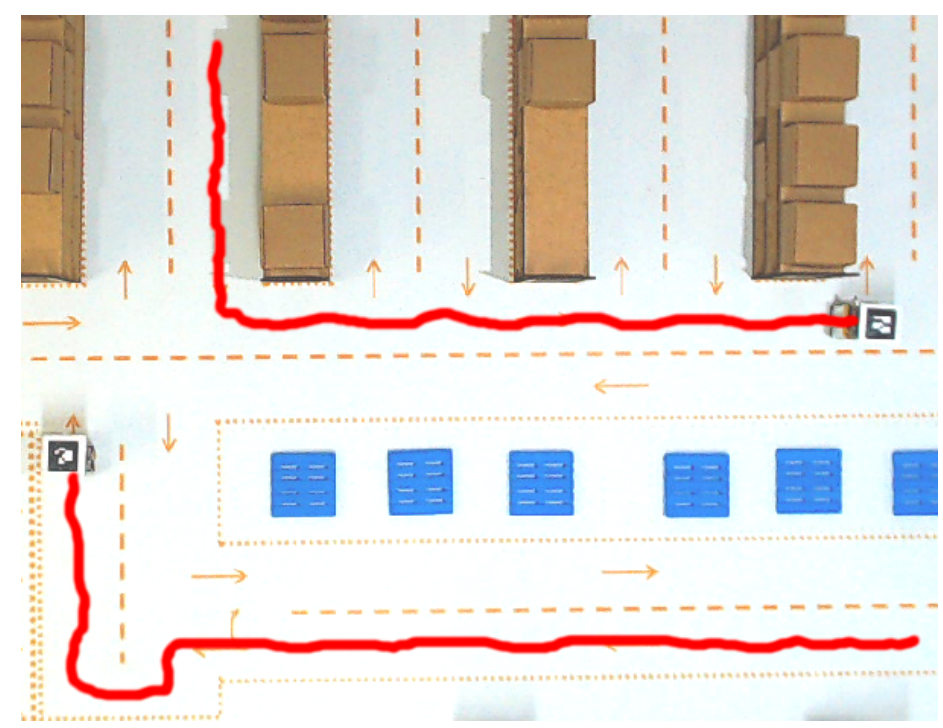

Fonte: Autoria própria.

cidade com que o sistema é capaz de atualizar essas coordenadas, enquanto o movimento do robô é executado.

Outra possível análise se faz em relação ao software de controle do robô, cujo sistema de controle deve garantir que o robô percorra todos estes pontos, no menor tempo e com o menor desvio possível da rota estipulada, a partir dos pontos determinados no planejamento de trajetória. Estes fatores dependem, basicamente, de um algoritmo de controle eficiente e um ajuste adequado de suas variáveis. Nas duas execuções é possível verificar pequenas oscilações no caminho executado. Essas oscilações podem ser causadas por uma associação significativa de fatores que, dificilmente, poderiam ser reproduzidos com precisão em um ambiente virtual. Além do atraso na determinação da posição do robô por meio do sistema de rastreamento, falhas no algoritmo de controle ou nos componentes do robô, falhas de comunicação, entre muitos outros. A execução desse experimento evidencia a vantagem em se adotar a arquitetura ROS e de seu caráter distribuído, pois desenvolver um sistema com essa quantidade de elementos, de software e de hardware, operando em sincronia seria, de fato, uma tarefa extremamente mais complexa sem as ferramentas e os recursos presentes no ROS.

\section{Conclusão}

Este trabalho apresentou a ARENA, uma plataforma híbrida (real e virtual) para experimentação de SMR em armazéns inteligentes. O fato de ter sido reproduzido um armazém real e toda a sua dinâmica de operação se mostrou eficaz para o desenvolvimento de soluções de logística em armazéns. Os elementos virtuais proporcionaram a implementação de uma série de características dinâmicas e complexas, que seriam inviáveis em função do tamanho reduzido da plataforma ou por questões orçamentárias. A visualização, em 
tempo real, de informações como os dados dos sensores ou da marcação do trajeto percorrido se mostraram muito úteis para a observação do comportamento do sistema, além de permitir o melhor entendimento dos estados das operações, que estão sendo realizadas. Essas informações podem ser personalizadas de acordo com as características de cada projeto desenvolvido, e poderiam, por exemplo, serem exibidas a carga de cada robô ou valores de variáveis internas, que serviriam de base para a tomada de decisões. A utilização de uma série de dispositivos reais como robôs, câmeras, dispositivos de rede entre outros, possibilitou o desenvolvimento de soluções que considerassem todas as limitações e imprecisões encontradas nesses componentes. Todas essas características tornaram a ARENA uma ferramenta valiosa para o desenvolvimento de diversas soluções com possibilidade de aplicação real e quase imediata.

\section{Referências}

Antoun, Valentini, Hocquard, Wiandt, Trianni, and Dorigo (2016). Kilogrid: A modular virtualization environment for the kilobot robot. IEEE/RSJ International Conference on Intelligent Robots and Systems (IROS), pp. 3809-3814, ieee edition.

Echeverria, G., Lemaignan, S., Degroote, A., Lacroix, S., Karg, M., Koch, P., Lesire, C., and Stinckwich, S. (2012). Simulating complex robotic scenarios with morse. In SIMPAR, pages 197-208.

Kagermann, H., Wahlster, W., and Helbig, J. (2013). Securing the future of german manufacturing industry. Recommendations for implementing the strategic initiative INDUSTRIE.

Kalempa, V. C., Piardi, L., Limeira, M., and De Oliveira, A. S. (2020). Faultresilient collective ternary-hierarchical behavior to smart factories. IEEE Access, 8:176905-176915.

Koenig, N. and Howard, A. (2004). Design and use paradigms for gazebo, an open-source multi-robot simulator. In 2004 IEEE/RSJ International Conference on Intelligent Robots and Systems (IROS)(IEEE Cat. No. 04CH37566), volume 3, pages 2149-2154. IEEE.

Lee, C. (2018). Development of an industrial internet of things (iiot) based smart robotic warehouse management system. In CONF-IRM 2018 Proceedings. 43.

Li, J.-t. and Liu, H.-j. (2016). Design optimization of amazon robotics. Automation, Control and Intelligent Systems, 4(2):48-52.

Limeira, M., Piardi, L., Cremer Kalempa, V., Schneider, A., and Leitão, P. (2020). Augmented Reality System for Multi-robot Experimentation in Warehouse Logistics, pages 319-330. 
Limeira, M., Piardi, L., Kalempa, V. C., Leitão, P., and De Oliveira, A. S. (2021). Depthlidar: Active segmentation of environment depth map into mobile sensors. IEEE Sensors Journal, pages 1-1.

Limeira, M. A., Piardi, L., Kalempa, V. C., de Oliveira, A. S., and Leitão, P. (2019). Wsbot: A tiny, low-cost swarm robot for experimentation on industry 4.0. In 2019 Latin American Robotics Symposium (LARS), 2019 Brazilian Symposium on Robotics (SBR) and 2019 Workshop on Robotics in Education (WRE), pages 293-298.

Mason, R. (2019). Developing a Profitable Online Grocery Logistics Business: Exploring Innovations in Ordering, Fulfilment, and Distribution at Ocado, pages 365-383.

Michel, O. (1998). Webots: Symbiosis between virtual and real mobile robots. In International Conference on Virtual Worlds, pages 254-263. Springer.

Piardi, L., Kalempa, V. C., Limeira, M., de Oliveira, A. S., and Leitão, P. (2019). Arena-augmented reality to enhanced experimentation in smart warehouses. Sensors, 19(19).

Pickem, D., Glotfelter, Wang, and Mote, M.; Ames, A. F. E. . E. M. (2017). The robotarium: A remotely accessible swarm robotics research testbed. IEEE International Conference on Robotics and Automation, 16.

Reina, Cope, Nikolaidis, Marshall, and Sabo (2017.). Ark: Augmented reality for kilobots. IEEE Robotics and Automation letters, vol. 2, no. 3, pp. 1755-1761 edition.

Reina, A., Salvaro, M., Francesca, G., Garattoni, L., Pinciroli, C., Dorigo, M., and IRIDIA, M. B. (2015). Augmented reality for robots: virtual sensing technology applied to a swarm of e-pucks. NASA/ESA Conference on Adaptive Hardware and Systems (AHS), Montreal, QC, Canada, 15417936 edition.

Rohmer, E., Singh, S. P., and Freese, M. (2013). V-REP: A versatile and scalable robot simulation framework. In 2013 IEEE/RSJ International Conference on Intelligent Robots and Systems, pages 1321-1326. IEEE.

ROS (2019a). ar_track_alvar ros wiki. http://wiki.ros.org/ar\_track _alvar. Last accessed 9 May 2019.

ROS (2019b). rviz ros wiki. http://wiki. ros.org/rviz. Last accessed 9 May 2019.

Zhang, X., Fronz, S., and Navab, N. (2002). Visual marker detection and decoding in ar systems: A comparative study. In Proceedings of the 1st International Symposium on Mixed and Augmented Reality, ISMAR '02, pages 97-, Washington, DC, USA. IEEE Computer Society. 